University of Nebraska - Lincoln

DigitalCommons@University of Nebraska - Lincoln

Faculty Publications, Department of Physics and Astronomy

Research Papers in Physics and Astronomy

2009

\title{
Voltage-induced switching with magnetoresistance signature in magnetic nano-filaments
}

\author{
Andrei Sokolov \\ University of Nebraska-Lincoln, sokolov@unl.edu \\ Renat F. Sabirianov \\ University of Nebraska - Omaha, rsabirianov@mail.unomaha.edu \\ Ildar F. Sabiryanov \\ University of Nebraska - Lincoln, isabirianov2@unl.edu \\ Bernard Doudin \\ University of Nebraska-Lincoln, bernard.doudin@ipcms.unistra.fr
}

Follow this and additional works at: https://digitalcommons.unl.edu/physicsfacpub

Part of the Physics Commons

Sokolov, Andrei; Sabirianov, Renat F.; Sabiryanov, Ildar F.; and Doudin, Bernard, "Voltage-induced switching with magnetoresistance signature in magnetic nano-filaments" (2009). Faculty Publications, Department of Physics and Astronomy. 94.

https://digitalcommons.unl.edu/physicsfacpub/94

This Article is brought to you for free and open access by the Research Papers in Physics and Astronomy at DigitalCommons@University of Nebraska - Lincoln. It has been accepted for inclusion in Faculty Publications, Department of Physics and Astronomy by an authorized administrator of DigitalCommons@University of Nebraska Lincoln. 


\title{
Voltage-induced switching with magnetoresistance signature in magnetic nano-filaments
}

\author{
A Sokolov ${ }^{1,2}$, R Sabirianov $^{2,3}$, I Sabirianov $^{1}$ and B Doudin ${ }^{4}$ \\ ${ }^{1}$ Department of Physics and Astronomy, University of Nebraska, Lincoln, NE 68588, USA \\ 2 Nebraska Center for Materials and Nanoscience, University of Nebraska, Lincoln, \\ NE 68588, USA \\ ${ }^{3}$ Department of Physics, University of Nebraska-Omaha, Omaha, NE 68588, USA \\ ${ }^{4}$ Institut de Physique et de Chimie des Matériaux de Strasbourg (IPCMS), \\ UMR 7504 CNRS-ULP, 67034 Strasbourg, France \\ E-mail: asokol@unlserve.unl.edu
}

Received 13 April 2009, in final form 9 September 2009

Published 30 October 2009

Online at stacks.iop.org/JPhysCM/21/485303

\begin{abstract}
Large hysteretic resistance changes are reported on sub-100 nm diameter metallic nanowires including thin dielectric junctions. Bi-stable 50\% switching in a double junction geometry is modeled in terms of an occupation-driven metal-insulator transition in one of the two junctions, using the generalized Poisson expressions of Oka and Nagaosa (2005 Phys. Rev.

Lett. 95 266403). It illustrates how a band bending scheme can be generalized for strongly correlated electron systems. The magnetic constituents of the nanowires provide a magnetoresistive signature of the two resistance states, confirming our model and enabling a four states device application.
\end{abstract}

(Some figures in this article are in colour only in the electronic version)

Hysteretic modification of the electrical resistance state under an applied high electric field has been observed for a variety of semiconductors $[2,3]$ and insulating $[4,5]$ materials. The quest for developing new permanent memory devices has renewed the interest in this topic, especially when seeking scaling-down and low-power usage. One particular family of devices involves transition metal oxides materials [6-9] sandwiched between metallic electrodes, i.e. metal-insulatormetal (M/I/M) structures involving strongly correlated electronic (SCEG) systems. A filamentary conductor several nanometers in diameter $[8,10,11]$ separated by thin interfacial layers [7, 12, 13] from the metallic electrodes is generally considered as the elemental source of resistance switching. There is a growing consensus that such a electro-resistive effect is related to the self-doping capability of transition metal oxides, which is promoted by local modulation of the oxygen content [8]. The origin of resistance switching remains, however, elusive. One fundamental reason is the complexity of the electric transport properties of such systems, owing to the strong electron-electron correlations, questioning for example the use of simple band structure arguments. Another reason is the limited ability to fabricate samples of adequate size, which should reconcile macroscopic electrical interconnects with the expected microscopic origin of the switching.

In this paper, we illustrate how a better insight into the phenomenon of resistance switching can be obtained by investigating one single conductive nanowire, of a size corresponding to the expected filamentary conductor size found in thick dielectrics, and incorporating insulating thin layers as the trigger for resistance change. We also combine electro-resistive and magnetoresistive properties by investigating magnetic nanowires [14, 15]. We investigate the tunnel magnetoresistance (TMR) signature of $\mathrm{M} / \mathrm{I} / \mathrm{M}$ structures, with spin dependent tunneling probabilities modified by a change of the relative magnetic orientations of the two magnetic electrodes [16].

Conductive filaments of diameter below $100 \mathrm{~nm}$ made of $\mathrm{Ni}$ and $\mathrm{Co}$ separated by non-conductive $\mathrm{NiO}_{x}$ barriers forming $\mathrm{Ni} / \mathrm{NiO} / \mathrm{Co} / \mathrm{Ni} / \mathrm{NiO} / \mathrm{Co}$ structure (figure 1 ) are obtained by template electrochemical synthesis, following our previous reports $[14,15]$. Polycarbonate track etch membranes with nearly perfect round pores, with a nominal pore size of $30 \mathrm{~nm}$, 

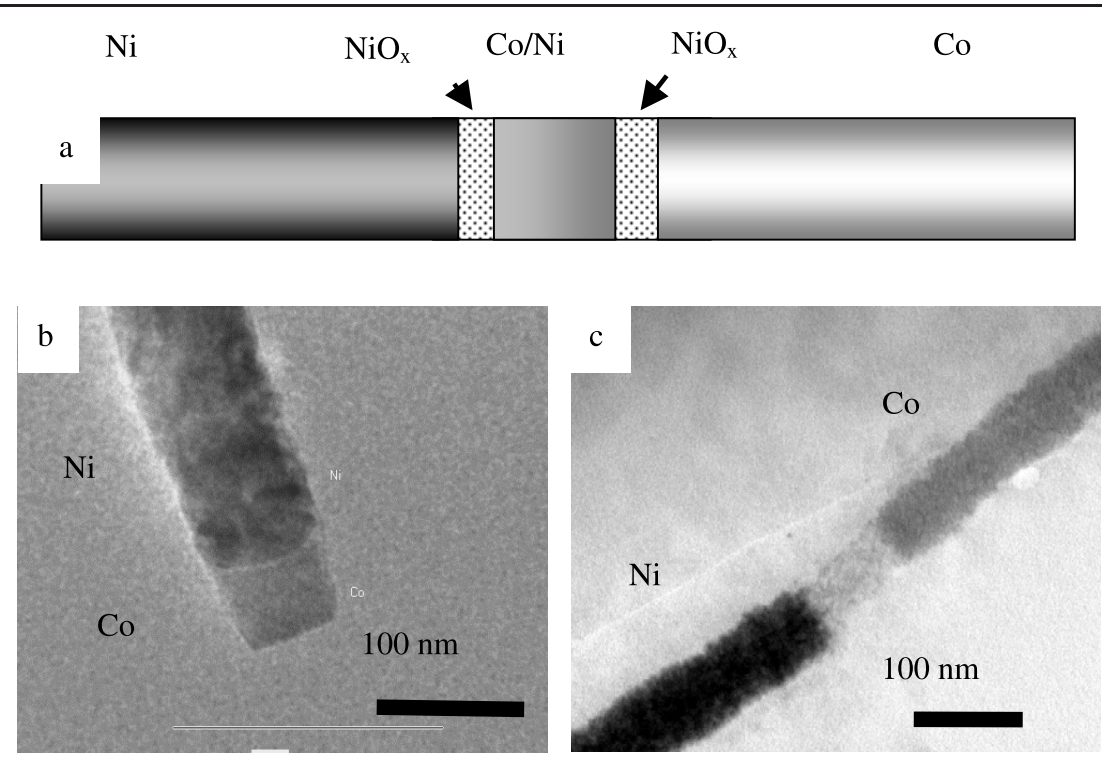

Figure 1. (a) Sketch of the double junction nanowire, involving successive growth of $\mathrm{Ni}$ (bottom electrode), $\mathrm{NiO}_{x}$ (first junction), Co followed by $\mathrm{Ni}$ (island), $\mathrm{NiO}_{x}$ (second junction), and $\mathrm{Co}$ (top electrode). Transmission electron micrograph (TEM) of the samples. (b) Ni-NiO-(CoNi), with a typical size of the $\mathrm{CoNi}$ island. (c) $\mathrm{Ni}-\mathrm{NiO}-\mathrm{NiCo}-\mathrm{NiO}-\mathrm{Co}$ nanowire with the central island showing very low contrast.

were used as templates. The metallic segments of nanowires filling the membrane were deposited in sequential order by replacing the bath containing $\mathrm{Ni}$ and $\mathrm{Co}$ ions, and applying an adequate deposition potential. Smooth and uniform $\mathrm{NiO}$ is obtained by anodization of the $\mathrm{Ni}$ top layers in $0.075 \mathrm{M}$ $\mathrm{Na}_{3} \mathrm{BO}_{3}$ and $0.3 \mathrm{M} \mathrm{H}_{2} \mathrm{~B}_{4} \mathrm{O}_{7}$. Characterization of the dielectric layer properties was made in situ by means of impedance spectroscopy. The estimated thickness is found to be about $1.5 \mathrm{~nm}$ [17]. Mott-Schottky analysis reveals the presence of ptype impurities with a concentration about $N_{a}=10^{25} \mathrm{~m}^{-3}$, somewhat lower than previously reported [18]. For TEM observation purposes, membranes filled with nanowires were dissolved in order to perform TEM analysis of the samples. The diameters of the nanowires were found to be in the range of $70 \pm 20 \mathrm{~nm}$, significantly larger than the nominal size of the membrane pores [19].

Nickel oxide is a Mott-type insulator that exhibits electroresistive switching properties $[9,11]$, often interpreted with a filamentary conduction model [20]. Our previous results on TMR single junctions in $\mathrm{Ni} / \mathrm{NiO} / \mathrm{Co}$ nanowires showed that filamentary conduction indeed occurred in those very small junctions [15]. Using tools developed for performing electrical connections to a single wire [21] we ensured that the investigated samples had sizes below $100 \mathrm{~nm}$ in diameter. Further reduction results in hot spots for the current passing through the $\mathrm{NiO}$ oxide. We previously observed that transport occurs within an area limited to a hot spot of a few nanometers size, for which single impurities were controlling the transport properties [14]. Extensive statistical studies indicated that impurity states in the barrier provide resonant tunneling paths, significantly affecting the TMR properties. The most significant outcome is the possibility to reverse the TMR sign [15].

No indications of resistance switching were found in more than $200 \mathrm{Ni} / \mathrm{NiO} / \mathrm{Co}$ single junctions samples, possibly due to their limited voltage stability, ultimately resulting in irreversible breakdown under a voltage bias typically exceeding $100 \mathrm{mV}$. We extended our study to double insulating barrier heterostructures $\mathrm{Ni}-\mathrm{NiO}-(\mathrm{CoNi})-\mathrm{NiO}-\mathrm{Co}$, obtained by incorporating a $\mathrm{CoNi}$ island by electrodeposition and repeating the anodization process for $\mathrm{NiO}$ fabrication. This middle segment was made by consecutive $\mathrm{Co}$ and $\mathrm{Ni}$ deposition, where Co plating from non-aqueous solution was necessary to keep the integrity of the insulating barrier, and $\mathrm{Ni}$ coating was essential for repeating the anodization process. Systematic electron microscopy imaging (figure 1) provided calibration of the thickness of the deposit as a function of the electric charge passed between working and counter-electrode. The smallest $\mathrm{Co}-\mathrm{Ni}$ thickness was $50 \mathrm{~nm}$, and all the results presented here are for islands of sizes between 50 and $150 \mathrm{~nm}$, which is the typical spread of the observed thickness under TEM observation. Samples made of longer islands showed results corresponding essentially to those observed for single junctions.

Hysteretic switching of the resistance value was observed at temperatures below $20 \mathrm{~K}$ on heterostructures with double $\mathrm{NiO}$ junctions (figure 2). The current value abruptly and irreversibly increases when the applied voltage reaches a threshold value of a few tens of $\mathrm{mV}$, at the onset of the low resistance (LR) state. The opposite potential of similar magnitude switches the system back to the high resistance (HR) state. The change of resistance values spanned magnitudes between $2 \%$ and $100 \%$, found on 26 samples (representing $20 \%$ success rate, also limited by breaking during cool-down or voltage sweeps). Nine samples showed reproducible behavior for more than ten voltage sweeps, without significant changes in the temperature range $1.5-20 \mathrm{~K}$. The magnetoresistance properties of the two resistance states (LR, HR) can be remarkably different, and are reminiscent of those found on single junction devices. The example of 

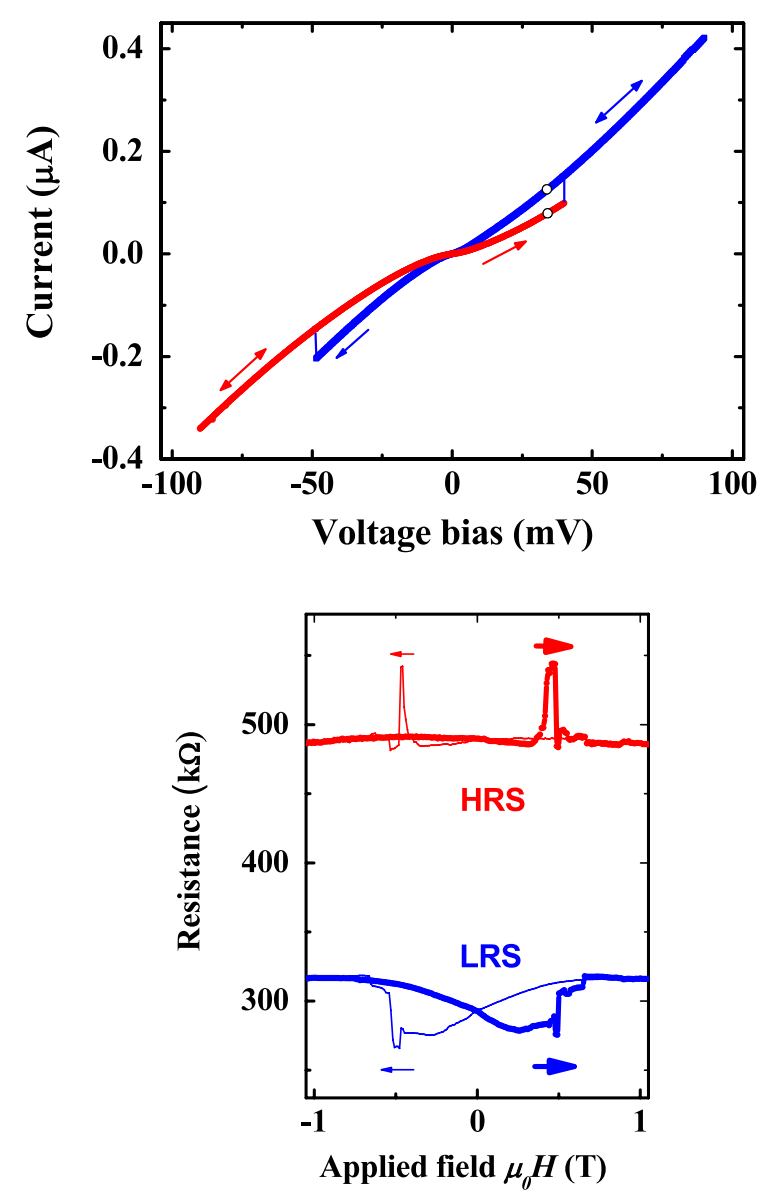

Figure 2. Top: IV curve of the $\mathrm{Ni}-\mathrm{NiO}-(\mathrm{CoNi})-\mathrm{NiO}-\mathrm{Co}$ double barrier nanowire, measured at $1.5 \mathrm{~K}$, showing reversible resistance switching. Bottom: magnetoresistance curves, under a DC bias held at $30 \mathrm{mV}$, of the two resistance states, with the color coding corresponding to the branches of the top figure.

figure 2 exhibits drastic differences in TMR properties when switching between HR and LR states. A model of currentinduced magnetization reversal mechanism [22] is unlikely to occur at the limited current densities we use, and cannot explain the data, since the magnetic-field-induced change has a sign and amplitude not corresponding to the voltage-induced change. Joule heating triggering the resistance switching was considered by several authors $[4,11,23]$. We took advantage of the sporadic occurrence of telegraph noise in single junction devices under significant potential bias [14] to perform local thermometry measurements [24]. The deduced heating of the junction did not exceed $10 \mathrm{~K}$ for current voltage products one order of magnitude larger than those reported here, allowing us to discard heating effects.

TMR properties of the HR and LR states differ in signs as well as the applied magnetic field values triggering resistance changes. The change of sign between the two magnetoresistance curves of figure 2 can be interpreted in terms of a formation/annihilation of a resonant state, providing a new preferred path for the electron to flow. We propose that one of the two junctions becomes conductive under an applied bias by means of the filling-driven Mott-Hubbard metal insulator (MI) transition model. This assumption explains the abruptness of the transition and its hysteretic nature. While stoichiometric nickel oxide with $\mathrm{Ni}^{2+}$ is an insulator, $\mathrm{NiO}_{x}$ with a large density of valence states can be brought very close to the metal-insulator transition. The $\mathrm{Ni}$ electrochemical anodization process is known to induce $\mathrm{Ni}^{2+}, \mathrm{Ni}^{3+}$ and even $\mathrm{Ni}^{4+}$ along with nickel vacancy states.

The occurrence of the MI transition in one of the barriers can be explained semi-quantitatively by using a simple carrier depletion/injection model. Figure 3 provides an intuitive qualitative picture of the process. When putting the p-type semiconductor $\mathrm{NiO}$ into equilibrium with the electrodes, a hole-rich region (figure $3(\mathrm{~b})$, left) appears at the positive metal/electrode interface and a hole-deficient region near the island promotes the insulating state. The applied voltage further depletes the barrier near to the negative electrode, enhancing its insulating state. In contrast, electrons attracted to the positive electrode leave behind available donor states and stimulate the appearance of the metallic state on the positive side. This simple band bending picture of figure 3 , based on Poisson's equation, remains remarkably valid for SCEG heterogeneous systems, even though a simple band structure description can become questionable. Oka and Nagaosa recently showed how density matrix renormalization group calculations can be used to map a modified Poisson's equation of the form [1]:

$$
\frac{\mathrm{d}^{2} V(x)}{\mathrm{d} x^{2}}=-\frac{e}{\varepsilon}\left(n(V(x))-n_{\mathrm{d}}\right)
$$

where $n(V(x))$ can be represented in a linearized form with constant compressibility $k=-\frac{\mathrm{d} n}{\mathrm{~d} V}=\frac{2}{W-\Delta}$ outside the Mott gap $\Delta$, while $n=1$ inside [25]. $W$ is the bandwidth. The concentration of carriers is described by the doping ratio $\delta$, i.e. impurity concentration $\left(N_{\delta}\right.$, per atomic concentration), related to bulk charge density $n_{\mathrm{d}}=1-\delta$. The equation is solved for $n$ with fixed $n_{\mathrm{d}}$. We assume pdoping for the barriers, following our Mott-Schottky analysis results. The much lower TEM contrast of the $\mathrm{Co} / \mathrm{Ni}$ metal island (figure 1) suggests high porosity of the island, that possibly has a significant amount of impurities (oxides most likely). We therefore arbitrarily choose a value significantly different from 1 for $n$ in the $\mathrm{Co} / \mathrm{Ni}$ metal island $(n=0.8)$. We use the midpoint technique with deferred corrections numerical method (Maple ${ }^{\mathrm{TM}}$ ) to solve Poisson's equation with the boundary conditions set by the applied voltage. The depletion layer width can be estimated from the full depletion analysis as $x_{\text {depletion }}=\sqrt{2 \varepsilon\left(V_{\mathrm{B}}-V_{\mathrm{A}}\right) / e N_{\delta}}$. Taking an impurity concentration of $N_{\delta}=2 \times 10^{25} \mathrm{~m}^{-3}, \varepsilon=4$ and the interface barrier $V_{\mathrm{B}}=\chi+\Delta-V_{\mathrm{M}}=0.5 \mathrm{~V}$ (a typical value, calculated as the difference of work function in the metal $V_{M}$, electron affinity $\chi$, and Mott gap $\Delta$ in SCEG), we estimate the depletion layer at zero applied potential to be about $3 \mathrm{~nm}$, i.e. larger than the width of our barrier, making it therefore insulating even though a significant doping ratio is present in our structures. Figure 3 illustrates the variation of the potential and charge density as a function of applied voltage across the double barrier structure. A short island length $(2 \mathrm{~nm})$ was chosen in order to calculate and draw the numerical results on a single scale. One should, however, note that identical 

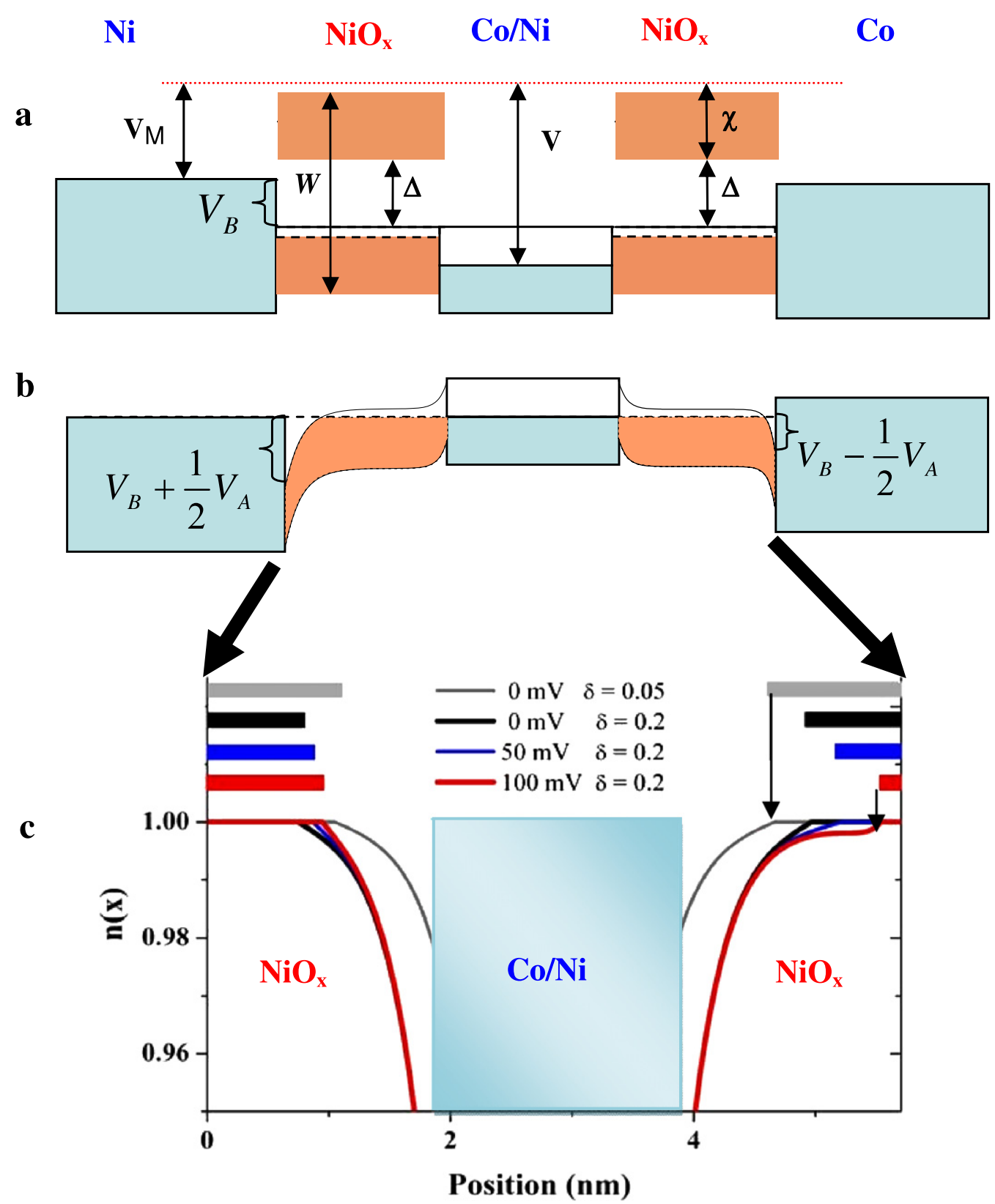

Figure 3. (a) Flat-band diagram and (b) band diagram under $V_{\mathrm{A}}$ applied voltage. $V_{\mathrm{B}}$ is the metal/semiconductor barrier height, $V_{\mathrm{M}}$ and $V_{\mathrm{I}}$ are the metal work functions of the electrodes and island, $\chi$ is the electron affinity, $\Delta$ is the gap of the band of width $W$. (c) Results of modified Poisson's equation calculation of the charge density and voltage in the heterostructure. Doping ratio is $\delta=0.0005$ inside the barriers, and $\delta=0.2$ in the island $n=1$ corresponds to insulating state while $n<1$ corresponds to metallic. Upper part demonstrates how increasing voltage difference $V_{\mathrm{A}}$ diminishes the insulating thickness of the barrier (bars) on the right side and becomes conducting at $100 \mathrm{mV}$. Lower part is the electron charge density $n(x)$ for several applied voltage values.

considerations and conclusions are drawn if the central island is longer. It is readily seen that the barrier at lower potential keeps the charge density essentially at $n(x)=1$, and, therefore its insulating property, under applied bias. The asymmetry comes from the interface at higher potential (left side of figure 3), where deviations from $n(x)=1$ promote a metallic state, corresponding to the nearly constant potential profile under applied bias, reaching the potential barrier between metal and semiconductor. The first order MI transition abruptly changes the potential profile across the device. The interfaces, essentially 3D systems, allow the filling-driven Mott transition to be of first order and to exhibit hysteresis, following the arguments given in [25].

The observed small value of the voltage threshold triggering the resistance switching is an important consequence of the double junction configuration. This finding, of key importance for device applications, can be explained by the asymmetry of the carrier density next to the two opposite electrodes. For a single barrier, the increasing carrier concentration on one side of the barrier that can promote the conducting state of the system is compensated by the depletion of carriers occurring on the other side 
of the barrier. In the double barrier geometry, the island breaks this inversion symmetry, and similarly bends the two barriers densities at the island/barrier interfaces (figure 3(b)). The carrier accumulations at one of the interfaces can add to the accumulations at one of the electrodes, therefore diminishing the insulator/metal switching voltage. We believe there is no physics prohibiting the MI transition in a single barrier structure [26], resulting in much larger amplitudes of resistance changes (but at the expense of an increased applied voltage). One should emphasize that the magnitude of resistance switching is not the key parameter for applications. Low-power applications require both resistance states of highenough magnitude, without huge variations resulting in an impedance mismatch in the circuitry.

By making artificial nanowire structures reproducing the filamentary origin of resistive switching, we emphasize the prospects of making double junction geometry devices for low-power applications, as well as a possibly richer device functionality obtained by creating hysteretic four resistance states attainable by magnetic and electrical excitations, analogous to observations on multiferroic junctions (keeping in mind the different mechanism) [5]. A simple electrostatic model, using a modified Poisson's equation, provides the quantitative tool explaining how a band bending picture explains our experimental findings. Although we applied this model to strongly correlated systems, other materials with filling-induced metal-insulator transition can be described similarly, in particular when invoking mechanisms of electromigration of vacancies with resulting local modifications of the oxygen content near interfaces [8].

\section{Acknowledgments}

This research was supported by Nebraska Research Initiative, NSF MRSEC (DMR 0820521), CHE-0518644. BD acknowledges support from a 'Chaire d'excellence' fellowship of the French ANR.

\section{References}

[1] Oka T and Nagaosa N 2005 Interfaces of correlated electron systems: proposed mechanism for colossal electroresistance Phys. Rev. Lett. 95266403

[2] Koshida N, Ueno K and Sheng X 1998 Field-induced functions of porous $\mathrm{Si}$ as a confined system J. Lumin. 8037

[3] Hovel H J and Urgell J J 1971 Switching and memory characteristics of $\mathrm{ZnSe}-\mathrm{Ge}$ heterojunctions J. Appl. Phys. 425076

[4] Rohde C, Choi B J, Jeong D S, Choi S, Zhao J-S and Hwang C S 2005 Identification of a determining parameter for resistive switching of $\mathrm{TiO}_{2}$ thin films Appl. Phys. Lett. 86262907

[5] Gajek M, Bibes M, Fusil S, Bouzehouane K, Fontcuberta J, Barthelemy A and Fert A 2007 Tunnel junctions with multiferroic barriers Nat. Mater. 6296

[6] Beck A, Bednorz J G, Gerber C, Rossel C and Widmer D 2000 Reproducible switching effect in thin oxide films for memory applications Appl. Phys. Lett. 77139

[7] Baikalov A, Wang Y Q, Shen B, Lorenz B, Tsui S, Sun Y Y, Xue Y Y and Chu C W 2003 Field-driven hysteretic and reversible resistive switch at the $\mathrm{Ag}-\mathrm{Pr}_{0.7} \mathrm{Ca}_{0.3} \mathrm{MnO}_{3}$ interface Appl. Phys. Lett. 83 957-9

[8] Szot K, Speier W, Bihlmayer G and Waser R 2006 Switching the electrical resistance of individual dislocations in single-crystalline $\mathrm{SrTiO}_{3}$ Nat. Mater. 5312

[9] Seo S, Lee M J, Seo D H, Jeoung E J, Suh D S, Joung Y S, Yoo I K, Hwang I R, Kim S H, Byun I S, Kim J S, Choi J S and Park B H 2004 Reproducible resistance switching in polycrystalline NiO films Appl. Phys. Lett. 855655

[10] Nakamura A, Matsunaga K, Tohma J, Yamamoto T and Ikuhara Y 2003 Conducting nanowires in insulating ceramics Nat. Mater. 2453

[11] Kim D C, Seo S, Ahn S E, Suh D S, Lee M J, Park B H, Yoo I K, Baek I G, Kim H J, Yim E K, Lee J E, Park S O, Kim H S, Chung U I, Moon J T and Ryu B I 2006 Electrical observations of filamentary conductions for the resistive memory switching in NiO films Appl. Phys. Lett. 88202102

[12] Ziese M, Bollero A, Panagiotopoulos I and Moutis N 2006 Magnetoresistance switch effect in a multiferroic $\mathrm{Fe}_{3} \mathrm{O}_{4} / \mathrm{BaTiO}_{3}$ bilayer Appl. Phys. Lett. 88212502

[13] Dietz G W, Antpohler W, Klee M and Waser R 1995 Electrode influence on the charge transport through $\mathrm{SrTiO}_{3}$ thin films J. Appl. Phys. 786113

[14] Doudin B, Redmond G, Gilbert S E and Ansermet J P 1997 Magnetoresistance governed by fluctuations in ultrasmall Ni/NiO/Co Junctions Phys. Rev. Lett. 79933

[15] Tsymbal E Y, Sokolov A, Sabirianov I F and Doudin B 2003 Resonant inversion of tunneling magnetoresistance Phys. Rev. Lett. 90186602

[16] Tsymbal E Y, Mryasov O N and LeClair P R 2003 Spin-dependent tunnelling in magnetic tunnel junctions J. Phys.: Condens. Matter $15 \mathrm{R} 10$

[17] Sokolov A, Jennings J R, Yang C-S, Redepenning J and Doudin B 2001 In situ characterization of ultra-small magnetic junctions made by electrochemical techniques MRS Proc. 674 T5.9

[18] Barral G, Njanjo-Eyoke F and Maximovitch S 1995 Characterisation of the passive layer and of hydroxide deposits of nickel by impedance spectroscopy Electrochim. Acta $\mathbf{4 0} 2815$

[19] Chlebny I, Doudin B and Ansermet J P 1993 Pore size distributions of nanoporous track-etched membranes Nanostruct. Mater. 2 637-42

[20] Jung K, Seo H, Kim Y, Im H, Hong J, Park J-W and Lee J-K 2007 Temperature dependence of high- and low-resistance bistable states in polycrystalline $\mathrm{NiO}$ films Appl. Phys. Lett. 90052104

[21] Wegrowe J E, Gilbert S E, Kelly D, Doudin B and Ansermet J P 1998 Anisotropic magnetoresistance as a probe of magnetization reversal in individual nano-sized nickel wires IEEE Trans. Magn. 34 903-5

[22] Ralph D C and Buhrman R A 2006 Concepts in Spin Electronics ed S Maekawa (Oxford: Oxford University Press) pp 195-238

[23] Song H, Tokunaga M, Imamori S, Tokunaga $Y$ and Tamegai T 2006 Nonvolatile multivalued memory effects in electronic phase-change manganites controlled by Joule heating Phys. Rev. B $\mathbf{7 4} 052404$

[24] Chun K and Birge N O 1994 Single-defect thermometer as a probe of electron heating in Bi Phys. Rev. B 492959

[25] Kotliar G, Murthy S and Rozenberg M J 2002 Compressibility divergence and the finite temperature Mott transition Phys. Rev. Lett. 89046401

[26] Bowen M, Maurice J L, Barthelemy A, Prod'homme P, Jacquet E, Contour J P, Imhoff D and Colliex C 2006 Bias-crafted magnetic tunnel junctions with bistable spin-dependent states Appl. Phys. Lett. 89103517 